\title{
ESTADO ACTUAL DEL PATRIMONIO PALEONTOLÓGICO DEL PERÚ
}

\author{
Vera Alleman ${ }^{1}$ \\ Sofía Benavente ${ }^{2}$
}

\begin{abstract}
RESUMEN
El objetivo de este trabajo fue el examinar la situación actual de los sitios fosilíferos y las colecciones de fósiles desde una visión global sobre la protección de este legado patrimonial. Los materiales fundamentales para el estudio, la investigación y la enseñanza de la paleontología están en las colecciones de fósiles y sus localidades. Hay colecciones privadas de aficionados interesados en la belleza de los fósiles o en el valor comercial de los mismos. Hay colecciones privadas científicas de investigadores activos que guardan su material de trabajo en su domicilio o en su centro de trabajo. Generalmente, las colecciones «tipo» están guardadas en un museo o un laboratorio universitario.

La situación actual de la que partimos, es que, no tenemos una legislación de los bienes paleontológicos. Se propone definir los restos paleontológicos y sus localidades como Patrimonio Natural Paleontológico y en consecuencia establecer un régimen tutelar particular; al igual que disfrutan otras entidades patrimoniales como el Arqueológico, el de Recursos Naturales y el Cultural.

Así mismo se muestran vistas de las colecciones de paleontología de las Universidades Ricardo Palma, la Universidad Nacional de San Agustín y la Universidad Nacional del Altiplano como un avance del trabajo paleontológico en las Universidades del Perú.
\end{abstract}

Palabras Clave: Colecciones, Patrimonios Naturales y Culturales, Universidad Ricardo Palma, Universidad Nacional de San Agustin, Universidad Nacional del Altiplano.

\section{SUMMARY.}

The objective of this work was to examine of the present situation of the fossil sites and a global vision the fossil collections on the protection of this patrimonial legacy. The fundamental materials for any paleontological study, the research and the paleontology education are in the collection of fossils and their localities. There are private collections that they are interested for fossil samples because it is beautiful or it has a commercial value. There are private scientific collections of active investigators who keep their material in home or its work center. Generally, the «type» collections are kept in a museum or a university laboratory. The present situation that we started off is that, we do not have a good paleontological legislation. We propose to define the paleontological samples and the localities as a like Paleontological Natural Patrimony and consequently to establish a particular regime tutelary; as enjoy other patrimonial organizations as like the Archaeological, the Natural Resources and the Cultural. Also we show pictures of the paleontology collections of the Ricardo Palm University, the San Agustín National University and the Altiplano National University as an advance of the paleontological work in the Universities of Peru.

Key Words: Colections, Natural and Cultural Patrimony , Universidad Ricardo Palma, Universidad Nacional de San Agustin, Universidad Nacional del Altiplano.

1 Universidad Ricardo Palma, Museo de Historia Natural. Apartado Postal 18-0131; e-mail: valleman@mail.urp.edu.pe

2 Facultad de Geología y Metalurgia - Laboratorio de Paleontología y Medio ambiente, Universidad del Altiplano e-mail: lube20@yahoo.com 


\section{INTRODUCCIÓN}

Los materiales fundamentales para el estudio, la investigación y la enseñanza de la paleontología, están en las colecciones fósiles y sus localidades fosilíferas. Hay colecciones privadas de investigadores activos que guardan su material de trabajo en su domicilio o en su centro de trabajo, que es, generalmente un museo o una universidad.

Existen varias universidades donde se imparten las carreras de geología y de biología , en la perspectiva de la formación académica del profesional que desea especializarse en paleontología.

En la Universidad Ricardo Palma, Facultad de Ciencias Biológicas, en la sección de Paleontología y con el apoyo del Consejo Nacional de Ciencia y Tecnología (CONCYTEC), a través de su Programa de Apoyo al Investigador, viene funcionando el Museo de Historia Natural, en el cual existen restos bien conservados de animales y de plantas, producto de las investigaciones realizadas desde 1985 por eminentes paleontólogos, biólogos y geólogos peruanos y extranjeros, acompañados por los estudiantes de la carrera de Biología. Esta colección cuenta con numerosos ejemplares de tipos, géneros nuevos y especies nuevas.

En la Universidad Nacional de San Agustín, Facultad de Geología, Geofísica y Minas, el Laboratorio de Paleontología, se cuenta con restos fósiles desde corales, braquiópodos, trilobites, briozoarios, lamelibranquios, equinodermos, hasta restos de vertebrados, producto de las investigaciones realizadas por egresados, paleontólogos y geólogos de esta universidad.

La Universidad Nacional del Altiplano , la Facultad de Geología y Metalurgia cuenta con el Gabinete de Paleontología, Micropaleontología y Medio Ambiente donde se guardan restos fósiles de trilobites, bivalvos, conularios, braquiópodos, gasterópodos y restos de plantas.
Es necesario tener una legislación de los bienes paleontológicos y de sus localidades. A la fecha solo se cuenta con la ley número 24047, Ley General de Amparo al Patrimonio Cultural y el D. L. número 22680 del 19.11.1979 donde se aprueba la Convención sobre las medidas que deben adoptarse para prohibir e impedir la importación, la exportación y la transferencia de propiedad ilícita de bienes culturales de la UNESCO, considerando el Art. 1 inc. a) Las colecciones y ejemplares raros de zoología , botánica, mineralogía, anatomía y los objetos de interés paleontológicos.

\section{OBJETIVOS}

1.- Evaluar la situación que presentan en la actualidad los sitios fosilíferos y las colecciones de las Universidades enmarcada dentro de un Patrimonio Natural Paleontológico.

2.- Dar a conocer el estado actual de los museos de la Universidades San Agustín de Arequipa, Ricardo Palma y la Nacional del Altiplano.

\section{ANTECEDENTES}

En el año 1995, a raíz del Seminario « Cien Años de Paleontología en el Perú " , realizado por la Academia Nacional de Ciencia y Tecnología del Perú (ANCYT), se concluye la necesidad de que las universidades peruanas deben estar actualizadas en la formación profesional con especialización en paleontología, consistente en:

a) Constituir un cuerpo organizado a nivel nacional donde estén registradas las instituciones que poseen colecciones científicas de fósiles.

b) Cada una de las instituciones deberá hacer un registro de los ejemplares que están en sus colecciones y elaborar un banco de datos con clasificación por taxonomía, localidad, edad y otras observaciones pertinentes.

c) Elaborar una red informática y publicar catálogos.

A comienzos de septiembre de 1997, más de 100 paleontólogos que representaban a 30 países, se reunieron en el Museo 
Senckenberg, Frankfurt, Alemania, para participar en la Conferencia Mundial sobre « Paleontología en el Siglo 21 «. La reunión representó un foro para identificar y discutir diferentes aspectos que conciernen a la Paleontología y a sus diversos ramas. Como objetivo final se aceptó la necesidad de iniciar y nutrir un diálogo que concierne al futuro de la Paleontología con una llamada a una acción meditada de todos los paleontólogos del mundo acerca de las colecciones y sus inventarios.

A la fecha $¿$ Cuanto se ha avanzado, en legislación ?. Si atendemos al origen y naturaleza de los restos paleontológicos fósiles y yacimientos, su protección debería enmarcarse dentro de un Patrimonio Natural Paleontológico, ya que se trata de objetos naturales, no creados por la acción del hombre. Sin embargo, se debe considerar que es constitutivo de estos objetos, y de la propia ciencia que los estudia, su dimensión histórica y su pertenencia a un periodo determinado de la historia de la tierra.

\section{CONCEPCIÓN DE PATRIMONIO PALEONTOLÓGICO}

De acuerda a Barrera Rodríguez (1990), todo aquel bien, mueble o inmueble, que posee un interés paleontológico, será considerado integrante del Patrimonio Paleontológico. Según Morales (1996), quién distingue entre objetos paleontológicos, los que integren un inventario o catálogo, y patrimonio paleontológico, que se seleccionarían, según criterios objetivos por el determinados, de los que componen ese inventario o catálogo.

También hay que tener en cuenta la coincidencia de valores paleontológicos con otros valores naturales (Formaciones geológicas, ecosistemas, etc.). La gestión de los yacimientos paleontológicos englobados en Formaciones o Zonas con otros valores naturales de importancia, no es un problema de índole conceptual sino competencial y administrativo.
En la actualidad, en el ámbito de Canarias, los bienes paleontológicos son regulados por la ley 16/1985 de 25 de Junio, del Patrimonio Histórico Español y contemplados en la ley 12/1994 de 19 de Diciembre.

En España, se cuenta con la ley de Patrimonio Histórico Español (1985) y la ley de Espacios Naturales Protegidos (1989).

En el Perú, se cuenta con la Ley número 24047 Ley General de Amparo al Patrimonio Cultural y el Decreto Legislativo número22680 del 19.11.1979, donde se aprueba la Convención sobre las medidas que deben adoptarse para prohibir e impedir la importación, la exportación y la transferencia de propiedad ilícita de bienes culturales de la UNESCO, en el Art. 1 inc. A). Las colecciones y ejemplares raros de zoología, botánica, mineralogía, anatomía y los objetos de interés paleontológicos.

\section{ESTADO ACTUAL DE LAS COLECCIONES DE FÓSILES PERUANOS.}

Primero y ante todo, tenemos que estar conscientes que ninguna institución podrá jamás tener una colección completamente suficiente para la formación académica de sus alumnos, y esto tampoco es necesario. De estos dos hechos resulta que es preciso organizar la colaboración entre instituciones. Lo que es indispensable, y está a nuestro alcance hacerlo, es que se organice una eficiente coordinación en la documentación, en la infraestructura, en la accesibilidad a las muestras y en el apoyo mutuo entre las diferentes instituciones educativas y empresariales. Esta coordinación debería realizarse a nivel nacional.

Es de prioridad uno: La documentación referente a las colecciones. La primera pregunta que el estudiante se hace es «¿Dónde están las colecciones de consulta?», seguida inmediatamente de esta pregunta» ¿Qué hay en esas colecciones?». Para responder a esas preguntas, se necesita elaborar un registro de las instituciones que disponen de colecciones 
científicas de fósiles, con el contenido de las colecciones. Se aconseja elaborar un banco de datos a nivel nacional, computarizado e interconectado, en una especie de red consultiva, de tal manera que el usuario disponga de un máximo de información referente al fósil, de su ubicación y del estado de conservación en que se encuentra.

Esta organización podría también ser muy útil para la consulta empresarial . Imagínese un paleontólogo en función en una empresa o un geólogo de campo que desea confirmar su diagnóstico de una amonita descrita por Carlos Lissón. Con la computadora, en el campo mismo, en conexión con el registro, este profesional podría inmediatamente saber donde comparar su ejemplar y, tal vez, si estamos bien organizados, recibir una imagen por scanner y otros datos necesarios al diagnóstico referente a los ejemplares que están a su alcance en un determinado centro. Entonces, el interesado decidirá si vale o no la pena desplazarse para una consulta personal.

Por supuesto para llevar a cabo este tipo de proyecto de gran eficiencia y calidad se necesitará el concurso de varios especialistas paleontólogos y de diversas orientaciones dentro de esta ciencia.

Eso, a su vez, implica la existencia de universidades peruanas actualizadas en la formación profesional con especialización en paleontología.

En resumen, realizar esta primera etapa de un programa de desarrollo de Paleontología es un proceso bastante realizable:

a) Se constituye un cuerpo organizado a nivel nacional en el que quedan registradas las instituciones que poseen colecciones científicas de fósiles.

b) Cada una de las instituciones hace un registro de los ejemplares que están en sus colecciones y elabora un banco de datos con clasificaciones por taxonomía, localidad, edad y otras observaciones útiles como, fauna y flora asociadas, etc...

c) Se elabora una red informativa.

d) Paralelamente se publican los catálogos.
Una vez ordenada de este modo la colección nacional, es necesario el mantenimiento de las colecciones en buenas condiciones de conservación.

Para eso se requiere adquirir una infraestructura adaptada, en un ambiente adecuado. Por ejemplo, la humedad de Lima destroza el material fosilífero. La manipulación de los fósiles frágiles también debe evitarse. En el caso de fósiles comunes hay que prever el reemplazo, y si se trata de material difícil de conseguir, es mejor proporcionar moldes de yeso o de plástico a los estudiantes.

Mantener las colecciones implica también un compromiso de complementarlas en forma planificada, armoniosa $y$ paralelamente, con los nuevos trabajos de exploración, de estudio y de investigación por parte de las empresas y universidades. En esta etapa también se necesitará una buena cantidad de profesionales paleontólogos.

Una vez que están ordenadas, la documentación fosilífera, la infraestructura y los ambientes para la conservación, se puede pasar a la etapa de desarrollo de la accesibilidad de consulta y de estudio de las colecciones.

Es necesario de adecuar un ambiente de estudio y el apoyo y dedicación de una persona que atienda a los estudiantes. Puede tratarse, provisionalmente, de un estudiante avanzado, que realice esta tarea como práctica preprofesional bajo la supervisión de un paleontólogo experimentado.

La última tarea, la más importante y la más especializada a cumplir para estar al día con nuestras colecciones, es la actualización en cronología y en taxonomía. ¿Cuántos están registrados con edades incorrectas ? ¿Cuántos fósiles tienen una identificación inadecuada, fuente de errores y malentendidos? ¿Cuántos fósiles deben ser estudiados en forma complementaria y figuren «mientras tanto» con un « cf.» o un «aff.», cuando en realidad se trata de nuevos taxones?. En esta etapa es recomendable iniciar el trabajo con los 
fósiles tipos y los fósiles guías. Muchos de estos están en peligro de desaparecer con su nombre peruano, absorbidos e incorporados en modernos estudios de bioestadística y población en países vecinos. Necesitan ser descritos de nuevo, en una forma actualizada, y sus especies conocidas más profundamente por estudios bioestadísticos. En la mayoría de los casos se necesita hacer colecciones complementarias en localidades típicas, haciendo columnas estratigráficas y observaciones de campo en sedimentología y paleoecología.

Todos estos trabajos, tan necesarios, se están realizando en los países vecinos, desde décadas y requieren personal de formación universitaria altamente calificada.

La Universidad Ricardo Palma dispone de una sección de Paleobiología en su Museo de Historia Natural. En aquella sección están depositadas varias colecciones de tipos, colecciones de tesis de título de licenciatura de Biología, colecciones privadas, colecciones de intercambio internacionales, colecciones extranjeras y colecciones de investigación. Dentro de las colecciones de investigación se cuentan las muestras de los doctores Víctor Benavides, Hermann Pfefferkorn, Thomas De Vries, Luc Ortlieb, y de los biólogos Aldo Indacochea, Nelly Vargas, Carmen Castro, Pedro Tapia y la suscrita. Por supuesto tiene también sus colecciones de enseñanza, de didáctica que desde que se inició el dictado del curso de Paleontología, en 1969, fueron parcialmente en forma generosa proporcionadas por la profesora de Paleontología de la Universidad Nacional de Ingeniería, la doctora Rosalvina Rivera, y por el entonces profesor de Paleontología de la Universidad Nacional Mayor de San Marcos, el Ingeniero Guillermo Morales. Y por terminar, además, dispone de una original colección de etnopaleontología.

También tiene colecciones únicas de fichas de tipos de fósiles peruanos ilustradas, con las descripciones del francés, inglés, latín y neerlandés, traducidas al castellano.
La Universidad Nacional de San Agustín cuenta con su laboratorio de Paleontología donde se guardan restos fósiles desde corales, braquiópodos, trilobites, briozoarios, lamelibranquios, equinodermos hasta restos de vertebrados, producto de las investigaciones realizadas por el doctor Juan Ortiz, la Ing. Vilma Garcia y geólogos egresados.

Así mismo la Universidad Nacional del Altiplano cuenta con el Gabinete de Paleontología, Micropaleontología y Medio Ambiente, donde se encuentran restos fósiles de trilobites, bivalvos, conularios, braquiópodos, gasterópodos y restos de plantas, como resultado de las investigaciones iniciadas en la década del 80 por el Dr. Kurt Grove Froverg, el ingeniero Newton Machaca, algunas tesis de los egresados y la suscrita.

\section{CONCLUSIONES}

1.- Es necesario establecer un régimen tutelar particular al igual del que disfrutan otras entidades patrimoniales, como por ejemplo el Patrimonio Arqueológico, Patrimonio Natural y el Patrimonio Cultural..

2.- La firma de Convenios entre Universidades nacionales y extranjeras es de vital importancia.

3.- Dar inicio a trabajos interdisciplinarios y orientados al análisis entre la vida y la tierra a través del tiempo geológico.

4.- Siendo los paleontólogos los custodios de la vida y que nutren nuestro conocimiento sobre el valor que la misma tuvo en la historia de la tierra es necesario propiciar una ley enmarcada dentro del Patrimonio Paleontológico.

\section{REFERENCIAS BIBLIOGRÁFICAS}

ALLEMAN, V. (1985) Paleontología de los Carbones Paleozoicos Peruanos. Primer Symposium Nacional del Carbón, Lima 1985, Fasc. 5: 12 pp. Universidad Nacional de Ingeniería, Lima. 
ALLEMAN, V. (1994) Los Trigoniidae de la Colección Víctor Benavides, depositados en la URP. Biotempo, 1, URP: 27 - 29. Universidad Ricardo Palma, Lima.

ALLEMAN, V. (1995) El Estado de la Taxonomía y de las Colecciones de Fósiles Peruanos en Seminario «Cien Años de Paleontología en el Perú. Actas ANCYT, 3 (2): 1 - 5. Academia Nacional de Ciencia y Tecnología, Lima.

ARCHANGELSKY, S. (1997) Paleontología en el Siglo 21. Noticias, 5 ( 1-2 ). Asociación Latinoamericana de Paleobotánica y Palinología, Lima.

BARRERA, R. (1990) La Ordenación jurídica del Patrimonio histórico. 735 pp. Ed. Cevita, Madrid.

BENAVENTE, S. (1978) Reconocimiento paleontológico de las Calizas Arcurquina en el Paraje Canihuayo Cerro Pajonal. Tesis Bachiller en Geología. Universidad Nacional San Agustín, Arequipa.

BENAVENTE, S. (1986) Fósiles del Devoniano Cabanillas. Resúmenes Campo de Acción de la Ingeniería geológica. Universidad Nacional del Altiplano, Puno.

BENAVENTE, S. y C. ALVAREZ (2000) Aportes de la Paleobotánica en la

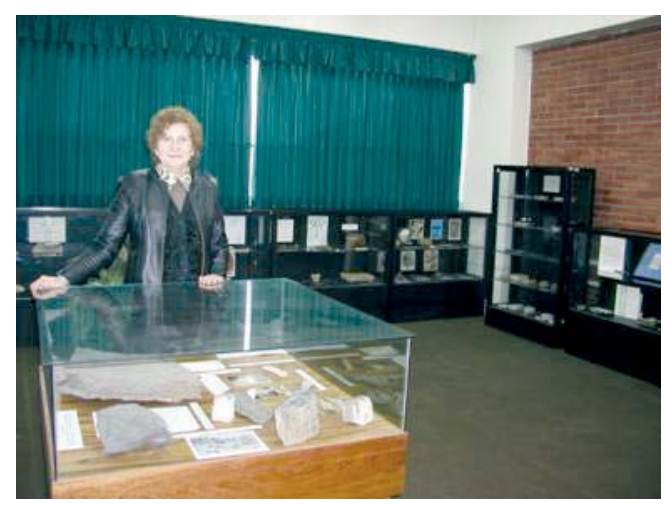

Fotografía 1: Museo de Historia Natural: La sala de la exposición didáctica de Paleontología, presentada por la autora.
Reconstrucción paleoclimática: 199. X Congreso Peruano de Geología. Resúmenes. Sociedad geológica del Perú, Lima.

CASTILLO, C. y cols. (1999) La Tutelar del Patrimonio Paleontológico en Canarias. Coloquios de Paleontología, 50: 9 - 21. Universidad Complutense, Madrid.

MELÉNDEZ, G. y cols. (1999) La Comisión de Patrimonio de la Sociedad Española de Paleontología (SEP): antecedentes, constitución y objetivos. Coloquios de Paleontología, 50:9-21. Universidad Complutense, Madrid.

MORALES, J. y cols. (1999) El Patrimonio Paleontológico Español. Coloquios de Paleontología, 50: 53 - 62. Universidad Complutense, Madrid.

UNIVERSIDAD NACIONAL DEL ALTIPLANO (1996) Diagnóstico de Funcionamiento de los Laboratorios y Gabinetes. Informe Memoria.

Velez, A. (1996) Estudio Bioestratigráfico del Potencial petrolífero de la Cuenca del Titicaca. Tesis de Bachiller. Universidad Nacional del Altiplano, Puno.

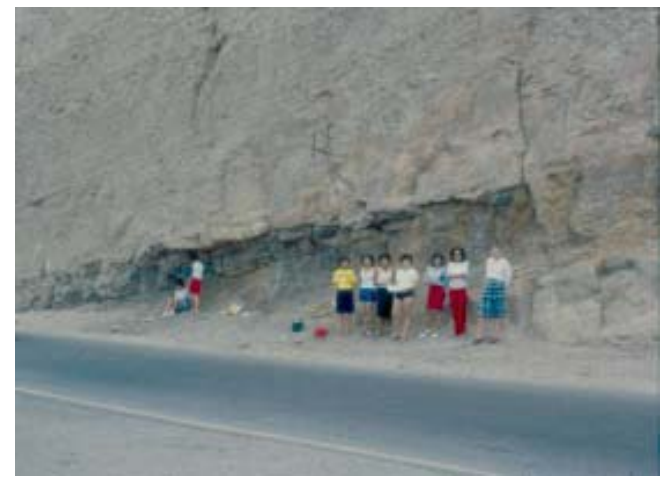

Fotografía 2: Alumnos del curso de Paleontología y la profesora Vera Alleman, realizando las prácticas en la formación Salto de Fraile, Moro Solar - Chorrillos, Lima. 